\title{
Identification of long non-coding RNAs that stimulate cell survival in bladder cancer
}

\author{
Aleksandra M. Dudek ${ }^{1}$, Sabrina J. Boer ${ }^{1}$, Nanda Boon ${ }^{1}$, J. Alfred Witjes ${ }^{1}$, Lambertus \\ A.L.M. Kiemeney ${ }^{1,2}$, Gerald W. Verhaegh ${ }^{1}$ \\ ${ }^{1}$ Department of Urology, Radboud University Medical Center, Radboud Institute for Molecular Life Sciences, Nijmegen, The \\ Netherlands \\ ${ }^{2}$ Department for Health Evidence, Radboud University Medical Center, Radboud Institute for Health Sciences, Nijmegen, The \\ Netherlands
}

Correspondence to: Gerald W. Verhaegh, email: Gerald.Verhaegh@radboudumc.nl

Keywords: long non-coding RNA, bladder cancer, MiTranscriptome, IncRNA, biomarker

Received: December 14, $2016 \quad$ Accepted: March 08, 2017 Published: March 16, 2017

Copyright: Dudek et al. This is an open-access article distributed under the terms of the Creative Commons Attribution License (CC-BY), which permits unrestricted use, distribution, and reproduction in any medium, provided the original author and source are credited.

\section{ABSTRACT}

For many years, research on the biology underlying bladder cancer focused on protein-coding genes which cover only about $3 \%$ of the human genome. Recently, it was discovered that a large part of the human genome is actively transcribed as long non-coding RNAs (IncRNAs). LncRNAs are master regulators of gene expression and several IncRNAs were shown to play a role in bladder cancer development and progression. Here, we analyzed IncRNA expression in muscle-invasive bladder cancer (MIBC) using the MiTranscriptome database of cancer IncRNA expression profiles, and we studied their function in bladder cancer-derived tumor cells. Analysis of the MiTranscriptome IncRNA expression data revealed four MIBC subgroups, which partially overlapped with the four mRNA clusters identified by The Cancer Genome Atlas consortium. Up-regulation of three IncRNAs CAT266, CAT1297, and CAT1647 in bladder cancer, in comparison to normal urothelium, was confirmed in an independent series of normal, non-muscle invasive (NMIBC) and MIBC tissue samples. Furthermore, expression levels of CAT1297 were found to be correlated with disease-free and overall survival in MIBC. Knockdown of CAT266, CAT1297, and CAT1647 decreased cell viability and colony formation, due to the induction of apoptosis. In conclusion, our data show that IncRNAs expression is de-regulated in MIBC and three aberrantly expressed transcripts regulate proliferation and apoptosis. Our data indicate that IncRNAs play an important role in MIBC development and progression and are a treasure chest for the discovery of new biomarkers.

\section{INTRODUCTION}

Bladder cancer is the most common malignancy of the urinary tract worldwide. Accurate diagnostic and prognostic biomarkers and effective treatments are lacking and represent major challenges in urological oncology [1]. Bladder cancer is a heterogeneous disease. Approximately 75 to $85 \%$ of patients are diagnosed with non-muscle invasive bladder cancer (NMIBC), which is characterized by a high recurrence rate (ranging from 30 to $80 \%$ in five years). Due to extensive follow-up and treatment of NMIBC the cost per patient is highest of all cancers [2]. The remaining group of patients ( 15 to $25 \%)$, present with muscle-invasive bladder cancer (MIBC) that often progresses to metastatic disease. 5-year survival after a diagnosis of MIBC is only approximately $50 \%[3]$.

For many years, research on the biology underlying bladder cancer focused on protein-coding genes [1]. Protein-coding sequences cover only about 3\% of the human genome [4]. However, a large part of the human genome is actively transcribed and most of the transcriptional output is represented by non-coding RNAs (ncRNAs). One class of ncRNAs are the long non-coding RNAs (lncRNAs), which are transcripts of more than 200 nucleotides lacking protein-coding potential $[4,5]$. Recently, a systematic analysis of all publicly available next generation sequencing data revealed that over $60 \%$ of genes expressed in normal and tumor tissues are in fact 
lncRNAs and that their expression is often lineage and even cancer-specific [5].

LncRNAs are key regulators of gene expression, acting both in the nucleus and in the cytoplasm. Nuclear lncRNAs influence gene expression in cis and trans by the recruitment of chromatin modifiers and interaction with transcription factors. LncRNAs located in the cytoplasm affect gene expression post-transcriptionally by modulating mRNA translation, stability or decay. Furthermore, IncRNAs may act as microRNA sponges, modulating the cellular levels of microRNAs and, hence, expression of their target genes [6].

Cancer development is associated with deregulation in gene expression, and aberrant lncRNA expression was shown to be implicated in the development of many different cancer types [7]. Also in bladder cancer several oncogenic and tumor suppressive lncRNAs have been identified, such as H19, MALAT1, MEG3, SNHG16, TUG1 and UCA1 [8]. LncRNA expression levels often correlate with prognosis and metastasis formation $[9,10]$ or occurrence of therapy resistance [11]. In bladder cancer, the up-regulation of UCAI was found to induce EMT, tumor cell migration and invasion [12] and it was shown to contribute to cisplatin and gemcitabine resistance [13]. Because of their significant role in cancer development lncRNAs might be potential targets for development of new therapies [14].

In the present study, we evaluated expression levels of lncRNAs in MIBC using the publicly available MiTranscriptome cancer IncRNA expression data [5]. Aberrant expression of three up-regulated transcripts (CAT266, CAT1297 and CAT1647) was confirmed in an independent series of normal urothelium, NMIBC and MIBC tissue samples. Antisense oligonucleotides were used to knockdown lncRNA expression in bladder cancer cell lines. We found that reduced expression of CAT266, CAT1297 and CAT1647 decreased cell viability and induced apoptosis. Our data suggest that lncRNAs play an important role in bladder cancer development and progression, indicating that MiTranscriptome lncRNAs expression data can be use as a platform for the discovery of new biomarkers and therapeutic targets for bladder cancer.

\section{RESULTS}

\section{Characterization of long non-coding RNAs expression in MIBC}

To determine the expression pattern of long noncoding RNAs in MIBC, we analyzed expression of 12,382 transcripts identified by Iyer et al. [5], who reanalyzed TCGA RNA-seq data. Heterogeneity of MIBC patients was revealed by hierarchical clustering of 2,504 deregulated lncRNAs into 4 distinct lncRNA expression clusters (cluster \#1-4, Figure 1A). Cluster 1 samples were characterized by the lowest overall expression of lncRNAs, cluster 2 and 3 samples showed intermediate levels of IncRNAs, whilst cluster 4 was characterized by overall the highest level of lncRNA expression (Figure 1B). Furthermore, IncRNA cluster 1 samples showed an overlap with TCGA cluster 2 and 3 (25.6 and 34.8\%, respectively) (Figure 1C), and showed higher KRT5, KRT6A and KRT14 mRNA expression, characteristic for basal/ squamous tumors (TCGA cluster 3) (Figure 1D). LncRNA cluster 2 was over-represented in papillary-like TCGA cluster 1 (79.2\% of samples, Figure $1 \mathrm{C})$, marked by high expression of $F G F R 3$, but also high expression of luminal differentiation markers GATA3 and FOXA1 (Figure 1D). LncRNA cluster 3 overlapped with TCGA cluster 2 and 4 (43.6 and $73.3 \%$ of samples) (Figure 1C) and showed high expression of immune-related genes $(C D 48, C D 37$ and CCL5) (Figure 1D). LncRNA cluster 4 was found to be overrepresented in TCGA cluster 1 and 3 (12.8 and $21.7 \%$ of samples) (Figure 1C), marked by higher FGFR3 expression, but in contrast to cluster 2 lower expression of GATA3 and FOXA1 (Figure 1D).

\section{CAT266, CAT1297 and CAT1647 are up-regulated in bladder cancer}

Of the 12,382 lncRNAs that are expressed in MIBC, 892 transcripts were found to be significantly downregulated in MIBC, whereas 724 transcripts were upregulated at least 2 -fold $(p<0.05$ unadjusted for multiple comparisons). We focused on up-regulated transcripts, as these are more likely to become implemented as a biomarker, and selected three aberrantly expressed transcripts (Figure 2A, Supplementary Table 3), namely CAT1297 and CAT1647 (the highest tumor/normal ratio based on average expression levels) and CAT266 (the highest tumor/normal ratio based on median expression levels). The up-regulation of these three lncRNAs in MIBC in comparison to normal urothelium was confirmed in an independent set of muscle-invasive bladder cancer and normal urothelium tissue samples, with at least 17 -fold increase in expression levels $(p<0.001, p<0.05$, $p<0.001$, resp.) (Figure 2B). CAT1647 was found to be MIBC-specific, whereas CAT266 and CAT1297 were also found to be up-regulated in NMIBC ( $p<0.05$ and $p<0.001$, resp.) (Figure 2B). In the majority of MIBC tumors $(90.16 \%$ in the MiTranscriptome cohort, and $95.92 \%$ in the Nijmegen validation cohort), at least one of the selected lncRNAs (CAT266, CAT1297, CAT1647) was up-regulated relative to the median expression levels of each IncRNA in normal urothelium (Figure 2C). Interestingly, up-regulation of CAT266 and CAT1297 was not associated with TCGA-identified MIBC subtypes, whereas expression of CAT1647 was found to be higher in the basal TCGA cluster 3 (Supplementary Figure 1).

Finally, we evaluated the expression of bladder cancer-associated lncRNAs that have been described in literature. Elevated expression of ANRIL [15], SChLAP1 
[16], HIF1A-AS2 [17], BC072678 [18], PCAT1 [19], TINCR [20], MALAT1 [21], TUG1 [22] in MIBC was confirmed in the MiTranscriptome analysis, however with much lower fold changes than was found for CAT266, CAT1297 and CAT1647 (Figure 2D, Supplementary Table 4).

\section{Association of IncRNA expression and tumor progression in the MiTranscriptome cohort}

Expression levels of CAT1647, CAT1297, and CAT266 were correlated with clinico-pathological parameters (Supplementary Table 5). Expression levels of CAT1647 were found to be significantly higher in high grade $(p=0.027)$ and $\mathrm{pT} 2$ tumors $(p=0.015)$, whereas expression of CAT1297 was significantly associated with male gender $(p=0.008)$. Furthermore, expression levels of CAT1297 significantly correlated with progression- free survival $(p<0.05)$ (Figure 2E) and overall survival $(p<0.01)$ (Figure 2F). Interestingly, despite the fact that CAT1297 is associated with MIBC development, patients expressing CAT1297 at higher levels showed significantly longer PFS and OS. No correlation of CAT1647 and CAT266 expression with PFS or OS was found. In multivariate analysis, CAT1297 expression remained an independent prognostic factor of MIBC overall survival (Supplementary Table 6).

\section{Tissue specific expression of MIBC-associated IncRNAs}

The MIBC-associated lncRNAs could serve as potential biomarkers to diagnose and monitor bladder cancer, using e.g. urine as an analyte. We evaluated their expression in other urological tissue types and tumors (also
A

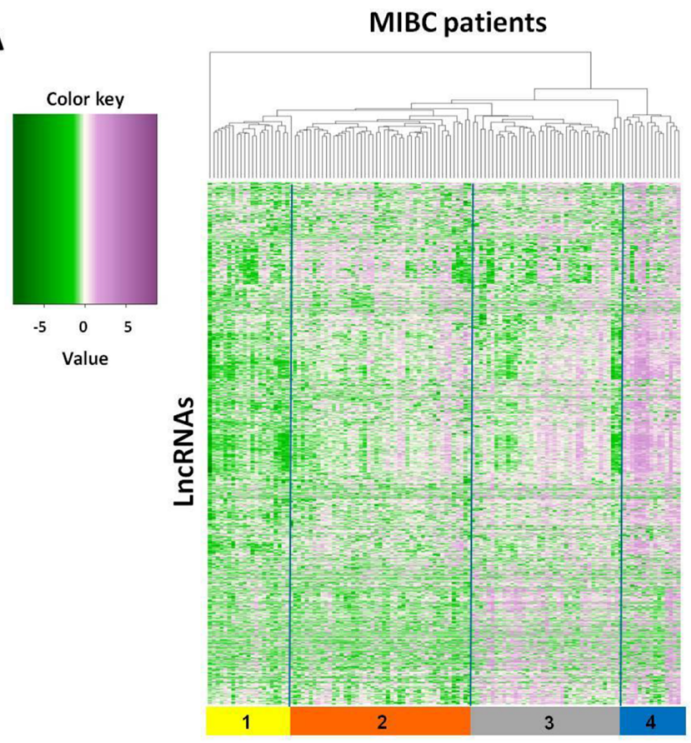

B

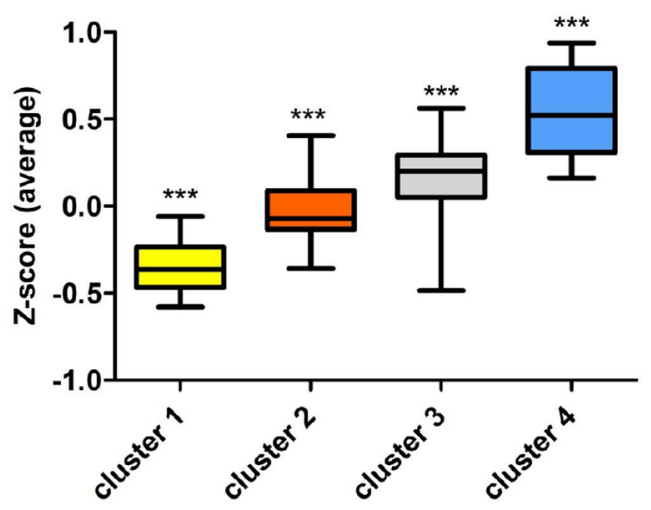

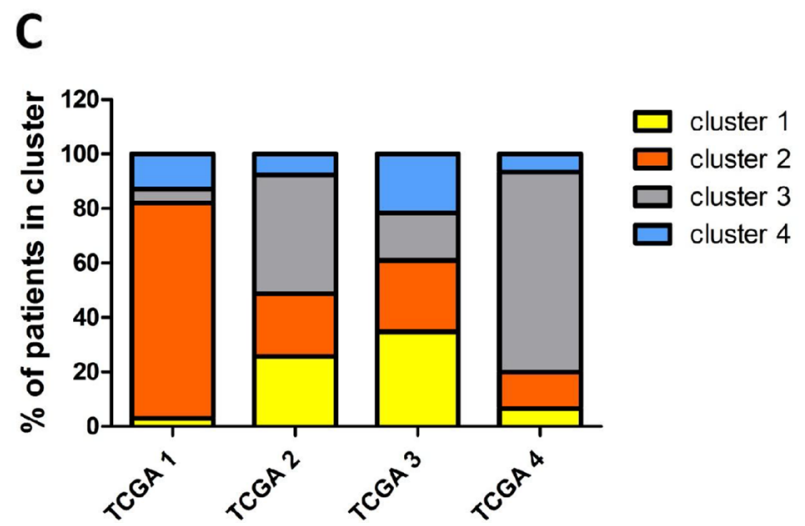

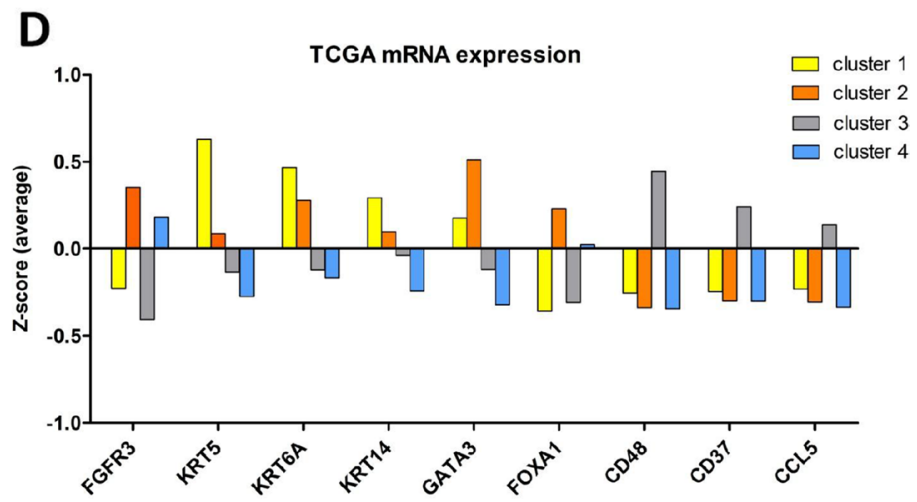

Figure 1: Characterization of IncRNAs expression in MIBC. (A) Hierarchical clustering analysis of lncRNA expression in 122 MIBC tissue samples identified 4 MIBC lncRNA clusters. FPKM data are log transformed and standardized by Z-score transformation. Green, negative Z-score; purple, positive Z-score. (B) The average expression levels (Z-score) of lncRNAs in the 4 identified clusters. Each cluster is characterized by significantly different average Z-scores $(p<0.001)$. (C) Overlap between 4 MIBC lncRNA clusters (cluster 1-4) and TCGA mRNA clusters (TCGA 1-4) [23]. (D) Expression levels of mRNAs defining TCGA clusters. The expression levels are shown as average $\mathrm{Z}$-scores in each of the lncRNA clusters. The bars represent mean. 
available in the TCGA project). Expression of CAT1297 was found to be significantly up-regulated in clear cell carcinoma and papillary cell carcinoma of the kidney compared to normal kidney $(p<0.001)$, and CAT266 was found to be up-regulated only in renal clear cell carcinoma $(p<0.05)$. No de-regulated expression of CAT1647 in different subtypes of kidney cancer was found. None of three MIBC-associated lncRNAs were found to be significantly deregulated in prostate cancer in comparison to normal prostate tissue. Most importantly, all three transcripts (CAT266, CAT1297 and CAT1647) are expressed at very low levels in normal kidney and normal prostate tissue (Figure 2G).

\section{Functional effects of $C A T 266, C A T 1297$ and CAT1647 knockdown}

To investigate the role of CAT266, CAT1297 and CAT1647, we used antisense oligonucleotides (ASOs) and siRNAs to knockdown their expression. Upon ASOmediated knockdown, significantly decreased expression levels of CAT266, CAT1297 and CAT1647 were found in comparison to control ASO-treated SW780 or SW800 cells (Figure 3A and Supplementary Figure 2, resp.). Reduced expression of CAT266, CAT1297 and CAT1647 significantly inhibited cell proliferation of SW780 (Figure 3B) and SW800 (Supplementary Figure 3) bladder cancer cells. SiRNA-mediated lncRNA knockdown in SW780 and SW800 bladder cancer cells was less efficient than ASO-mediated knockdown (Supplementary Figure 4). Consequently, the siRNA-mediated knockdown of CAT266 resulted in the decreased cell viability, however to a much lower extent than observed when using ASOs (Supplementary Figure 5). Colony forming assay confirmed that knockdown of CAT266, CAT1297 and CAT1647 impaired growth of cancer cells (Figure 3C), as indicated by a reduction in number of colonies (Figure 3D) and a decreased size of the colonies (Figure 3E). The reduced cell viability was found to be a result of induction of apoptosis marked by an increase in Caspase-3/7 activity (Figure 3F) and an increase in the sub-G1 cell population (Figure 3G). Finally, cell migration capacity was also inhibited when CAT1297 and CAT1647 were knocked down in SW780 cells (Figure 3H).

\section{Cellular localization of CAT266, CAT1297 and CAT1647}

LncRNAs are known to be master regulators of gene expression. They may regulate transcript levels preand post-transcriptionally, depending on their cellular localization [6]. In SW780 bladder cancer cells, CAT266 and CAT1647 were found to be significantly enriched in the cytoplasmic fraction, whereas similar levels of CAT1297 were found in both the cytoplasm and the nucleus (Figure 3I).

\section{DISCUSSION}

More than 330,000 patients are diagnosed with bladder cancer worldwide every year. Almost half of the MIBC patients, despite receiving treatment, progress to metastatic disease and eventually die within 5 years [3]. The Cancer Genome Project revealed that gene mutations, copy number alterations and de-regulated expression of protein-coding genes and microRNAs occur frequently in MIBC [23]. In our study we focused on non-proteincoding genes and by analysis of the MiTranscriptome lncRNA expression data [5] we identified numerous, aberrantly expressed transcripts in bladder cancer tissue.

De-regulated expression of IncRNAs has been shown to be associated with all canonical hallmarks of cancer, including regulation of proliferation, apoptosis, invasion, metastasis and angiogenesis [24]. Two studies showed that expression levels of multiple, bladder cancerspecific lncRNAs correlated with expression of proteincoding genes involved in the p53 signalling pathway, mTOR signalling or in cell cycle regulation [18, 25]. Peter et al. identified 32 lncRNAs that are associated with bladder tumor progression. Remarkably, the number of deregulated transcripts increased with invasive phenotype and, in contrast to our study, the majority of transcripts (91\%) were up-regulated. Knockdown of one of the identified lncRNA AB074278 led to decreased cell proliferation and induction of apoptosis [26]. Similarly, knockdown of CAT266, CAT1297 and CAT1647 activated apoptotic pathways leading to decrease in migration capacity, which is an important step for cancer cells allowing them to invade and eventually form metastasis [27]. This indicates that muscle-invasive tumors are directly dependent on lncRNAs expression for sustaining growth, cell survival and cell invasion.

Furthermore, the importance of lncRNA expression in MIBC is supported by the correlation of CAT1297 expression levels with progression-free and overall survival. Although up-regulation of CAT1297 was shown to be associated with MIBC cell survival, high CAT1297 levels correlated with longer overall survival. Currently, MIBC tumors are removed by cystectomy, and patients may receive cisplatin-based neo-adjuvant or adjuvant chemotherapy. The use of neo-adjuvant chemotherapy significantly increases OS [3]. As many lncRNAs have been shown to modify drug sensitivity [28, 29], it can be hypothesized that cells expressing CAT1297 are more susceptible to chemotherapy. One of the limitations of our study is the lack of sufficient clinical data about treatment, therefore correlation of lncRNA expression with chemotherapy response could not be assessed. Several markers predicting chemotherapy response have been identified, including DNA repair gene alterations [30] or mRNA and protein gene signatures [31]. Discovery of lncRNAs predicting response to treatment and survival, will allow for patient stratification and improvement of MIBC clinical management. 
A

MiT ranscriptome

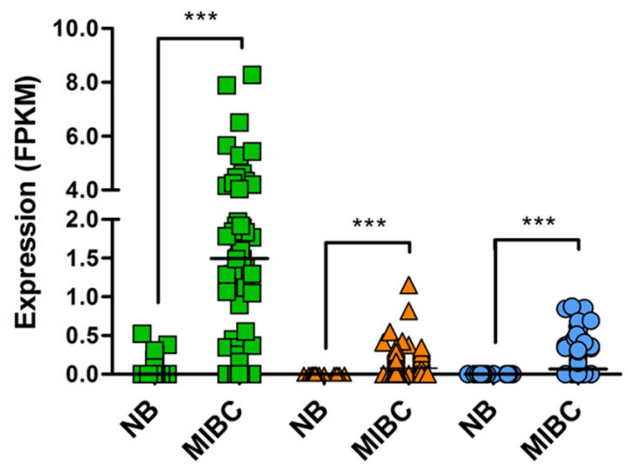

B

Validation

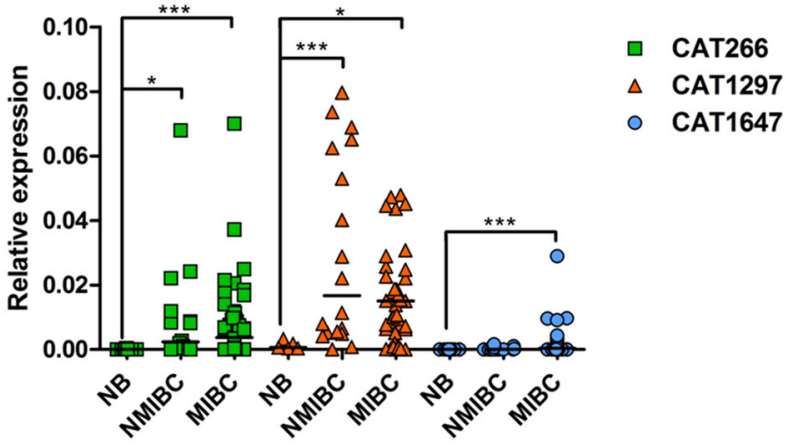

C

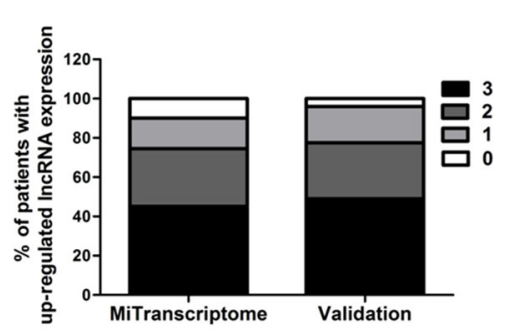

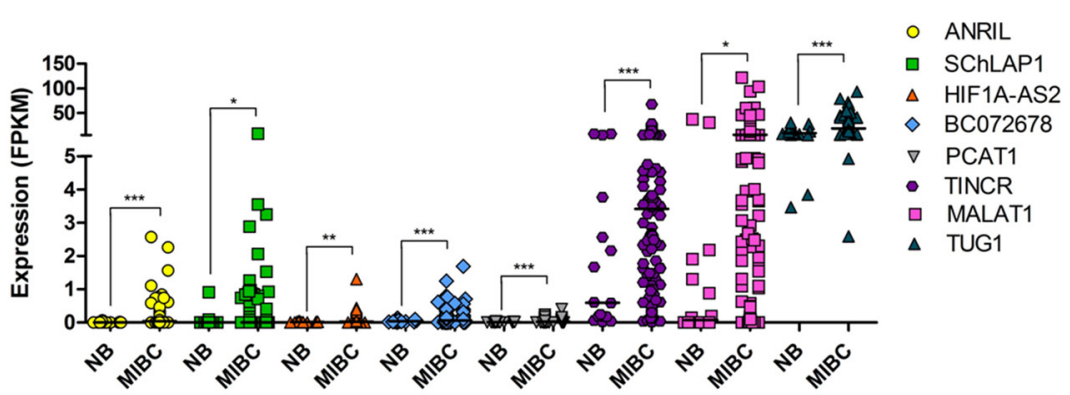

E

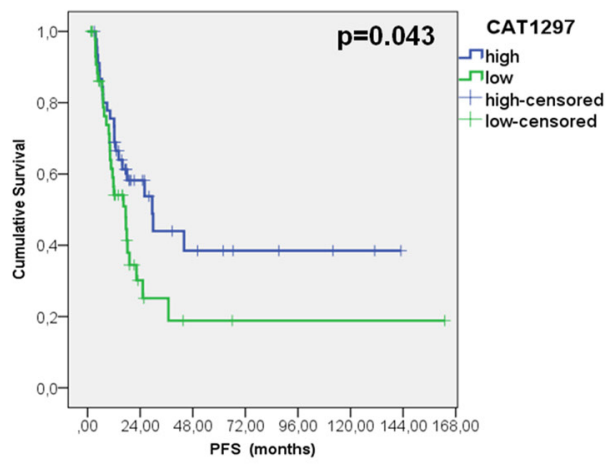

Number at risk

months

$C A T 1297$ high
CAT1297 low

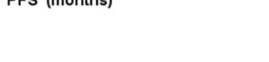

$$
\begin{array}{llll}
\mathbf{1 2} & \mathbf{2 4} & \mathbf{4 8} & \mathbf{7 2} \\
\mathbf{3 4} & 15 & 7 & 4 \\
\mathbf{2 2} & 6 & 1 & 1
\end{array}
$$

F

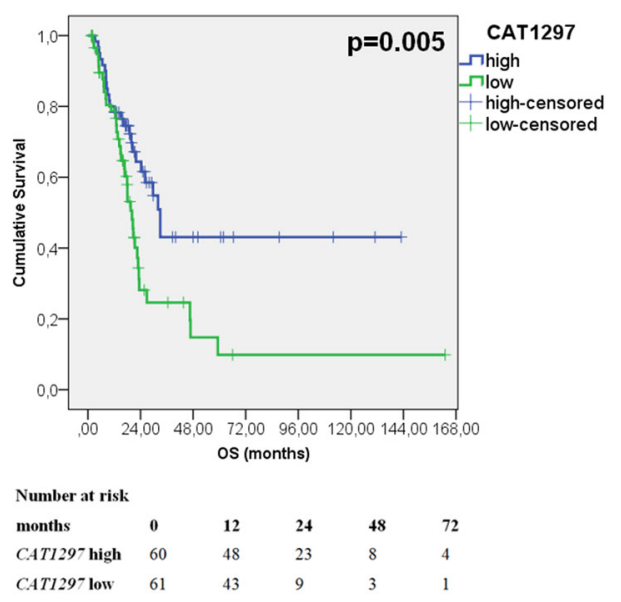

G

MiTranscriptome

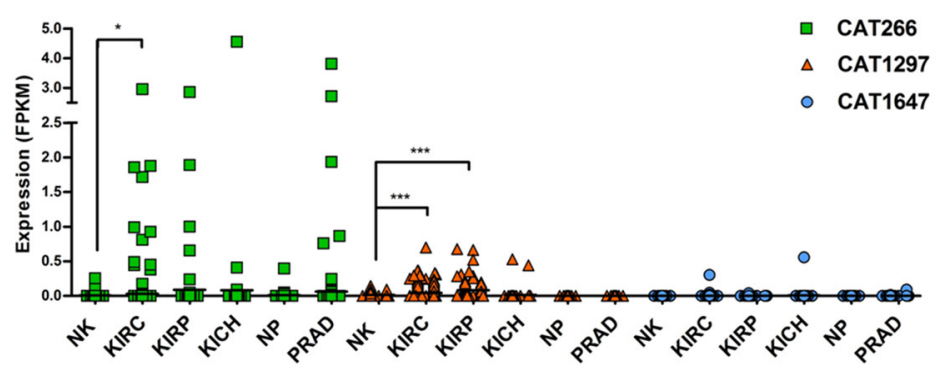

Figure 2: Expression of CAT266, CAT1297 and CAT1647 in normal urothelium (NB), non-muscle-invasive (NMIBC) and muscleinvasive bladder cancer (MIBC) in (A) MiTranscriptome discovery set and (B) in the Nijmegen validation set. Expression data were analyzed using unpaired $t$-test $(* p<0.05 ; * p<0.01 ; * * *<0.001$, line represent the median). (C) Percentage of MIBC patients with 3, 2, 1, 0 up-regulated lncRNA in the MiTranscriptome discovery set and in the validation set in comparison to median expression levels 
of each lncRNA in normal urothelium. (D) Expression levels of lncRNAs described as up-regulated in bladder cancer in literature, in the MiTranscriptome cohort of MIBC. Expression data were analyzed using unpaired $t$-test $\left({ }^{*} p<0.05 ; * *^{*}<0.01 ; * * * p<0.001\right.$, line represent the median). (E) Kaplan-Meier analysis, using data from the MiTranscriptome cohort, showing correlation of CAT1297 expression levels (high $n=46$; low $n=46$ ) with progression-free survival (PFS) and (F) overall survival (OS; high $n=60$; low $n=61$ ). (G) The expression levels of CAT266, CAT1297 and CAT1647 in other urological tissue types: normal kidney (NK, $n=119)$, kidney renal clear cell carcinoma (KIRC, $n=463$ ), kidney renal papillary cell carcinoma (KIRP, $n=77$ ), kidney chromophobe renal cell carcinoma (KICH, $n=64)$, normal prostate $(\mathrm{NP}, n=43)$ and prostate adenocarcinoma (PRAD, $n=174)$ in the MiTranscriptome dataset. Expression data were analyzed using unpaired $t$-test $(* p<0.05 ; * * p<0.01 ; * * p<0.001$, line represent the median).

NMIBC is characterized by high recurrence rate, leading to extensive follow-up of bladder cancer patients by cystoscopy. Urine is an ideal, non-invasive source for biomarker analysis, however, until now, no urinary marker is used in the clinic [2]. Two of the identified lncRNAs, CAT266 and CAT1297, were found to be significantly up-regulated not only in MIBC but also in a subset of NMIBC. Furthermore, expression of CAT266 and CAT1297 was found to be cancer-specific as they were not expressed, or at low levels, in normal urothelium, normal prostate and normal kidney tissue. Therefore, the potential use of the two identified markers, and in addition other de-regulated lncRNAs that may be identified using the MiTranscriptome database, for non-invasive bladder cancer monitoring should be further evaluated in a larger set of NMIBC tissue samples and in urine collected from NMIBC patients.

Despite the overall low expression levels of lncRNAs in comparison to protein-coding mRNAs, they are able to regulate a variety of cellular processes [6]. CAT266 is a novel lncRNA discovered by the MiTranscriptome study, and it located in a region in which no lncRNAs have been annotated before. CAT1297 partially overlaps with a previously identified lncRNA, RP11-190J1.3 and CAT1647 with RP11-1103G16.1, but to our knowledge no data on CAT266, CAT1297 and CAT1647 function have been published so far. We have shown that CAT266, CAT1297 and CAT1647 are expressed in the cytoplasm and in the nucleus, suggesting that they modulate gene expression levels through different mechanisms. More and more data are becoming available about the role of lncRNAs in RNA-RNA and RNA-protein crosstalk. LncRNAs can act as competing endogenous RNAs (ceRNAs), by regulating other RNAs through binding of microRNAs $[32,33]$. We analyzed potential lncRNAmicroRNAs interactions using the online available DIANA [34] and NPinter tools [35]. Multiple microRNAs were predicted to bind to CAT1297 and CAT1647, but these findings require further experimental validation in bladder cancer cell line models.

In conclusion, our data show that lncRNAs play an important role in bladder cancer development and progression, by regulating processes crucial for tumorigenesis, including cell proliferation and apoptosis. Further studies should focus on the identification of genes regulated by these bladder cancer-associated IncRNAs, in order to elucidate novel pathways involved in bladder tumorigenesis. A better understanding of the biology underlying MIBC may lead to the development of new treatment options for this life-threatening disease.

\section{MATERIALS AND METHODS}

\section{Identification of MIBC-associated IncRNAs from the MiTranscriptome database}

LncRNAs expression data (FPKM values) for muscle-invasive bladder cancer and normal urothelium tissue samples were downloaded from the MiTranscriptome database (http://mitranscriptome.org) [5]. Transcriptome data for the 16 normal urothelium and 122 MIBC tissue samples were generated in The Cancer Genome Atlas (TCGA) project. After exclusion of duplicates, expression data for 12,382 lncRNAs remained. Subsequently, 345 transcripts that were neither expressed in normal nor in cancer samples were excluded. From the remaining 12,037 lncRNAs, the most de-regulated transcripts were selected based on fold change in expression in normal and tumor samples (calculated using both average and median expression values) and based on $p$-values lower than 0.001 (unpaired $t$-test, IBM SPSS Statistics 22).

\section{Hierarchical clustering}

Based on differences in expression levels between normal and MIBC tissue samples in the MiTranscriptome cohort (unpaired $t$-test, $p<0.1$, unadjusted for multiple comparisons) 2,504 lncRNAs were selected for hierarchical clustering analysis. DNA mutation, copy number alteration and mRNA expression data for the patients and controls (that were included in the MiTranscriptome dataset) were downloaded from cBioPortal [36, 37]. Gene expression levels are highly skewed, and therefore, RNA expression FPKM values were log transformed and standardized by Z-score transformation per gene. These data were then visualized in a heat map that was generated using the heatmap. 2 function from the gplots package in $\mathrm{R}$ (version 3.1.2).

\section{Cell culture}

SW780 (ATCC\# CRL-2169) and SW800 bladder cancer cell line [38] were grown in RPMI-1640 medium (Invitrogen) supplemented with 10\% fetal calf serum (Sigma-Aldrich, F7524) and L-glutamine. Cell lines were cultured in a humidified atmosphere at $37^{\circ} \mathrm{C}$ and $5 \% \mathrm{CO}_{2}$. SW780 and SW800 were tested for Mycoplasma and 

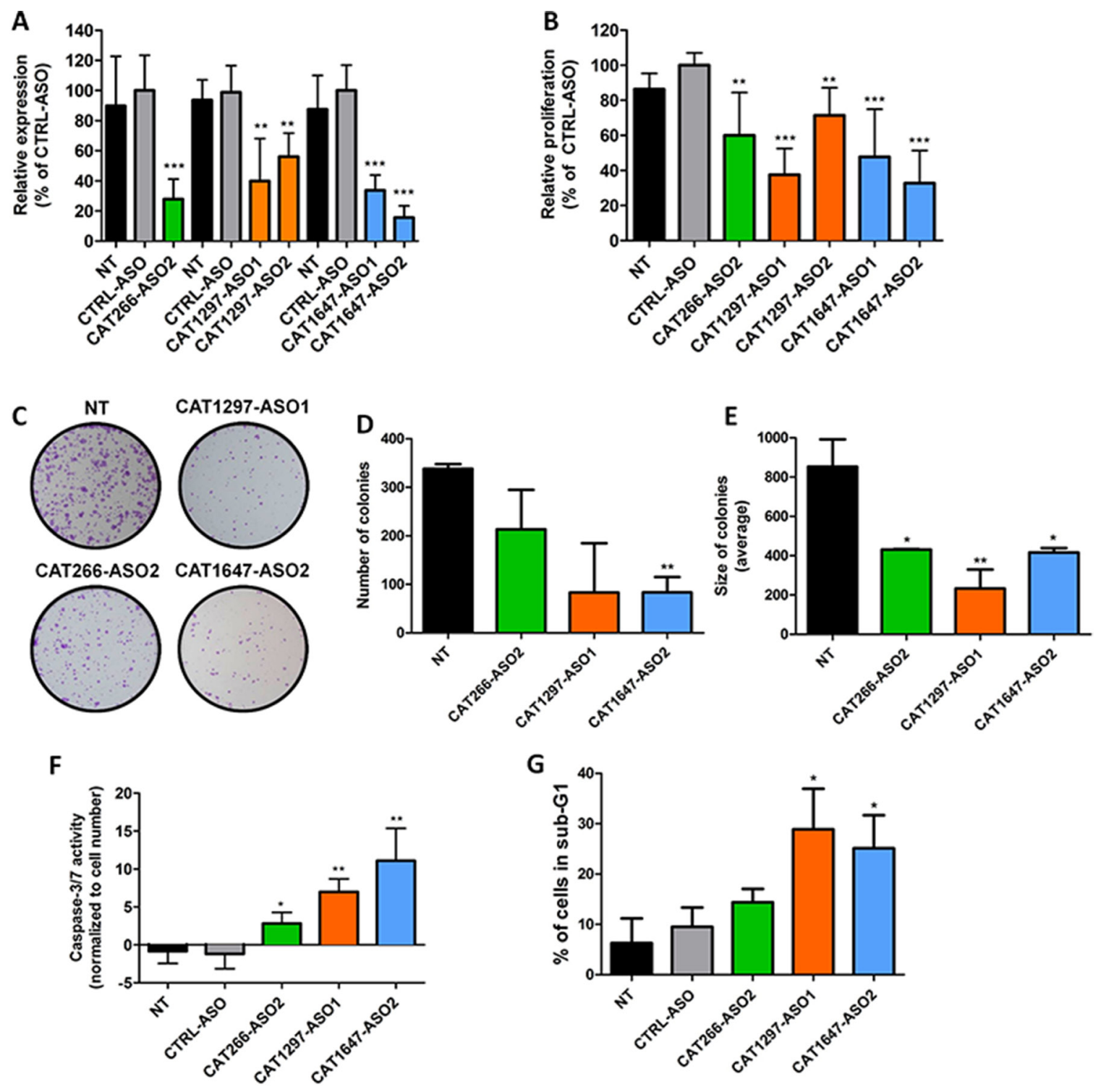

H

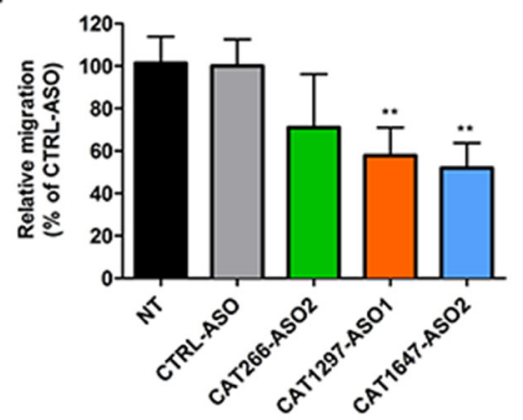

I

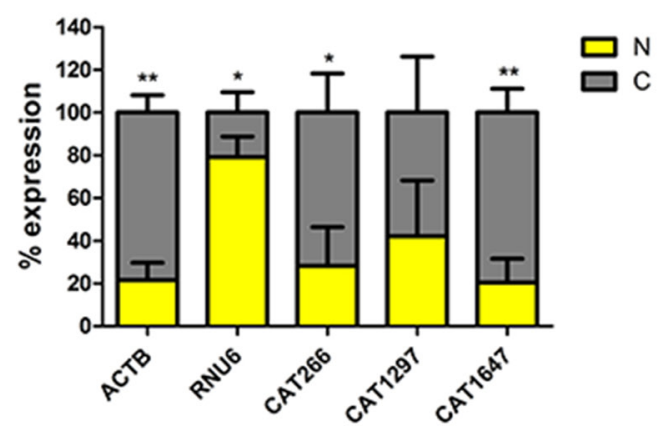

Figure 3: Knockdown of IncRNA expression. (A) Expression levels of CAT266, CAT1297 and CAT1647 (determined by RTqPCR) 48 hours after transfection of ASOs. The effect of CAT266, CAT1297 and CAT1647 ASO-mediated knockdown in SW780 cells on (B) cell proliferation, $(\mathbf{C})$ colony formation, (D) number of colonies and (E) size of colonies. (F) Induction of apoptosis upon knockdown of CAT266, CAT1297 and CAT1647, measured by an increase Caspase-3/7 activity. (G) Increase in sub-G1 cell population after knockdown of CAT266, CAT1297 and CAT1647, analyzed by propidium iodide staining and flow cytometry. (H) The effect of CAT266, CAT1297 and CAT1647 ASO-mediated knockdown in SW780 cells on migration. All data are compared to control ASO-transfected cells. ( $t$-test ${ }^{*} p<0.05 ; * p<0.01 ; * * p<0.001$, bars represent the mean $\left.\pm \mathrm{SD}\right)$. (I) Expression of CAT266, CAT1647 and CAT1297 in nuclear (N) and cytoplasmic (C) RNA fractions. ACTB (cytoplasmic RNA) and RNU6 (nuclear RNA) were used as controls for purity of isolated fractions ( $t$-test, ${ }^{*} p<0.05 ;{ }^{*} p<0.01$, bars represent the mean $\left.\pm \mathrm{SD}\right)$. 
authenticated in 2016 using the PowerPlex 21 PCR kit (Promega) by Eurofins Genomics.

\section{Antisense oligonucleotide- and siRNA-mediated knockdown of IncRNA expression}

Gapmer antisense oligonucleotides (ASOs) and small interfering RNAs (siRNAs) targeting up-regulated lncRNAs were designed using SFold software [39]. Twenty nucleotides long chimeric gapmers $\left(\mathrm{RNA}_{5}-\mathrm{DNA}_{10}-\mathrm{RNA}_{5}\right)$ were chemically modified by addition of phosphorothioate linkages and introduction of 2'-O-Me groups in the flanking RNA parts (Eurogentec). Custom Silencer siRNAs were used (Thermo Fisher Scientific). Antisense oligonucleotide and siRNA sequences are listed in Supplementary Table 1. Bladder cancer cell lines were transfected at $70-80 \%$ confluency with $0.7 \mu \mathrm{M}$ antisense oligonucleotide using X-tremeGENE 9 transfection reagent (Sigma-Aldrich) or with siRNAs at $20 \mathrm{nM}$ final concentration using Lipofectamine RNAiMAX transfection reagent (Thermo Fisher Scientific), according to the manufacturer's instructions. Each experiment was performed at least three times. Non-transfected cells were treated with transfection reagent only. All experiments are compared to control ASOs or control siRNA (Silencer Negative Control No.1 siRNA) transfected cells.

\section{Tissue collection and processing}

The use of patient materials was approved by the local ethics committee of the Radboud university medical center (CMO Arnhem-Nijmegen). Upon transurethral resection of tumor tissue (TUR) or cystectomy, specimens were snap frozen in liquid nitrogen. Normal urothelium (microdissected from bladder tissue obtained from bladder cancer patients after cystectomy, $n=5$ ), non-muscleinvasive bladder $(n=18)$, and muscle-invasive bladder cancer $(n=47)$ specimens were selected for purity of benign or cancer cells, respectively, and processed by step sectioning. Patient and tumor characteristics can be found in Supplementary Table 2.

\section{RNA isolation and RT-qPCR}

Total RNA was isolated using TRIzol reagent (Invitrogen) according to protocol. The RNA quantity and purity was evaluated using Nanodrop ND-1000 (Thermo Scientific). One microgram of RNA was DNaseI treated, and cDNA was synthesized using random hexamer primers and SuperScript II reverse transcriptase (Invitrogen). Gene expression levels were determined by SYBR Green qPCR (Roche) analysis in $16 \mu 1$ reaction volumes, performed in duplicate, using a LightCycler LC480 instrument (Roche). RNA not subjected to reverse transcriptase was used as a negative control for PCR amplification. Human Heterochromatin Protein 1, Binding Protein 3 (HP1BP3) was used for normalization and relative gene expression levels were quantified using the $\Delta \Delta \mathrm{Ct}$ method. Primer sequences are listed in Supplementary Table 1.

\section{Cell viability assay}

One day after transfection, cells were re-seeded into 96-well plates. Cell viability was assessed at regular time intervals using the CellTiter-Glo luminescence assay (Promega), according to the manufacturer's instructions. Luminescence was measured on the Victor ${ }^{3}$ multilabel reader (Perkin Elmer). Each experiment was performed in triplicate and repeated at least three times.

\section{Cell migration assay}

Cell migration was determined using a "wound healing" scratch assay. One day post transfection, cells were seeded in 6-well plates at high cell density and cultured to $100 \%$ confluence within 24 hours. The next day, scratches were made using a sterile pipette tip. Cells were subsequently rinsed with $0.9 \% \mathrm{NaCl}$, standard culture media was added, and cells were allowed to migrate into the cell-free area. Microscopic images were taken at regular time intervals after applying the scratch. The mean closure of the scratch was calculated from three individual scratches per group at each time point. Each experiment was repeated at least three times.

\section{Colony formation assay}

One day post transfection, cells were seeded in 6-well plates at a density of 500 cells/well, and cultured at standard conditions. After 12 days, colonies were washed with PBS, fixed with 3\% paraformaldehyde and stained with $0.01 \%$ crystal violet (Merck) for $30 \mathrm{~min}$. The number and size of colonies was analyzed using ImageJ software.

\section{Apoptosis assay}

One day post transfection, cells were seeded into 96-well plates. The next day, Caspase-3/7 activity was measured using the Apo-ONE Homogenous Caspase-3/7 Assay (Promega), according to the manufacturer's instructions. After $2 \mathrm{~h}$ of incubation, luminescence was measured on a Victor ${ }^{3}$ multilabel reader (Perkin Elmer). The luminescence signals were normalized to cell number (assessed by CellTiter-Glo luminescence assay) and Caspase-3/7 activity of non-transfected cells was used as background. Each experiment was performed in triplicate and repeated at least three times.

\section{Cell-cycle analysis}

One day post transfection, cells were seeded in 6-well plates. The next day cells were harvested, washed 
with $0.9 \% \mathrm{NaCl}$, resuspended in HBSS buffer (Invitrogen), and fixed with ice-cold ethanol. Fixed cells were centrifuged, resuspended in PBS and treated with RNase A $\left(1 \mathrm{mg} / \mathrm{ml}\right.$, Sigma) for $40 \mathrm{~min}$ at $37^{\circ} \mathrm{C}$. Subsequently, cells were stained with propidium iodide $(400 \mu \mathrm{g} / \mathrm{ml}$, Sigma) for $15 \mathrm{~min}$ in darkness. The samples were analyzed on a FC500 Flow Cytometer (Beckman-Coulter) and histograms were created using FlowJo software.

\section{Nuclear and cytoplasmic RNA isolation}

Nuclear and cytoplasmic RNA was isolated using the Cytoplasmic and Nuclear RNA Purification Kit (Norgen Biotek), according to the manufacturer's instructions. The RNA quantity and purity was measured on a Nanodrop ND-1000 (Thermo-scientific). The integrity of the RNA fractions was evaluated by $1 \%$ agarose gel electrophoresis. RNA was used for cDNA synthesis and qPCR analysis as described above. Primers specific for RNU6 (nuclear marker) and for $A C T B$ (cytoplasmic marker) were used to assess the quality of the RNA fractions (primer sequences are listed in Supplementary Table 1). Experiments were repeated three times.

\section{Statistical analysis}

All statistical analyses was performed using GraphPad Prism or IBM SPSS software, and $p<0.05$ was considered statistically significant. For lncRNA expression data, an unpaired $t$-test was used. In knockdown experiments, data were compared to control ASO-transfected cells or control siRNA-transfected cells and unpaired $t$-test was performed. Kaplan-Meier curves were analyzed using a Log-Rank (Mantel-Cox) test and Cox proportional hazard regression analyses were performed. Clinical data for bladder cancer patients included in the MiTranscriptome database were downloaded from cBioPortal $[36,37]$.

\section{Authors' contributions}

A.M.D, G.W.V and L.A.L.M.K designed the study. A.M.D and N.B performed the experiments and analyzed the data. S.J.B performed clustering analysis. A.J.W. provided patient material. A.M.D and G.W.V prepared the manuscript. S.J.B, A.J.W and L.A.L.M.K revised the manuscript.

\section{ACKNOWLEDGMENTS}

The results published here are in part based upon data generated by the TCGA Research Network (http:// cancergenome.nih.gov) and the MiTranscriptome catalog of long poly-adenylated RNA transcripts (http:// mitranscriptome.org). The authors would like to thank dr. Sita Vermeulen for statistical analysis of the survival data.

\section{CONFLICT OF INTEREST}

The authors declare no conflict of interest.

\section{FUNDING}

This research was sponsored by a research investment grant from the Radboud university medical center.

\section{REFERENCES}

1. Knowles MA, Hurst CD. Molecular biology of bladder cancer: new insights into pathogenesis and clinical diversity. Nature reviews Cancer. 2015; 15:25-41.

2. Babjuk M, Burger $M$, Zigeuner $R$, Shariat SF, van Rhijn BW, Comperat E, Sylvester RJ, Kaasinen E, Bohle A, Palou Redorta J, Roupret M, European Association of U. EAU guidelines on non-muscle-invasive urothelial carcinoma of the bladder: update 2013. European urology. 2013; 64:639-653.

3. Witjes JA, Comperat E, Cowan NC, De Santis M, Gakis G, Lebret T, Ribal MJ, Van der Heijden AG, Sherif A, European Association of U. EAU guidelines on muscleinvasive and metastatic bladder cancer: summary of the 2013 guidelines. European urology. 2014; 65:778-792.

4. Consortium Encode Project Consortium. An integrated encyclopedia of DNA elements in the human genome. Nature. 2012; 489:57-74.

5. Iyer MK, Niknafs YS, Malik R, Singhal U, Sahu A, Hosono Y, Barrette TR, Prensner JR, Evans JR, Zhao S, Poliakov A, Cao X, Dhanasekaran SM, et al. The landscape of long noncoding RNAs in the human transcriptome. Nature genetics. 2015; 47:199-208.

6. Fatica A, Bozzoni I. Long non-coding RNAs: new players in cell differentiation and development. Nat Rev Genet. 2014; 15:7-21.

7. Huarte M. The emerging role of lncRNAs in cancer. Nat Med. 2015; 21:1253-1261.

8. Martens-Uzunova ES, Bottcher R, Croce CM, Jenster G, Visakorpi T, Calin GA. Long noncoding RNA in prostate, bladder, and kidney cancer. European urology. 2014; 65:1140-1151.

9. Jadaliha M, Zong X, Malakar P, Ray T, Singh DK, Freier SM, Jensen T, Prasanth SG, Karni R, Ray PS, Prasanth KV. Functional and prognostic significance of long non-coding RNA MALAT1 as a metastasis driver in ER negative lymph node negative breast cancer. Oncotarget 2016; 7:40418-40436. doi: 10.18632/oncotarget.9622.

10. Prensner JR, Iyer MK, Sahu A, Asangani IA, Cao Q, Patel L, Vergara IA, Davicioni E, Erho N, Ghadessi M, Jenkins RB, Triche TJ, Malik R, et al. The long noncoding RNA SChLAP1 promotes aggressive prostate cancer and antagonizes the SWI/ SNF complex. Nature genetics. 2013; 45:1392-1398.

11. Teschendorff AE, Lee SH, Jones A, Fiegl H, Kalwa M, Wagner W, Chindera K, Evans I, Dubeau L, Orjalo A, 
Horlings HM, Niederreiter L, Kaser A, et al. HOTAIR and its surrogate DNA methylation signature indicate carboplatin resistance in ovarian cancer. Genome Med. 2015; 7:108.

12. Xue M, Pang H, Li X, Li H, Pan J, Chen W. Long noncoding RNA urothelial cancer-associated 1 promotes bladder cancer cell migration and invasion by way of the hsa-miR-145-ZEB1/2-FSCN1 pathway. Cancer science. 2016; 107:18-27.

13. Pan J, Li X, Wu W, Xue M, Hou H, Zhai W, Chen W. Long non-coding RNA UCA1 promotes cisplatin/gemcitabine resistance through CREB modulating miR-196a-5p in bladder cancer cells. Cancer letters. 2016; 382:64-76.

14. Leucci E, Vendramin R, Spinazzi M, Laurette P, Fiers M, Wouters J, Radaelli E, Eyckerman S, Leonelli C, Vanderheyden K, Rogiers A, Hermans E, Baatsen P, et al. Melanoma addiction to the long non-coding RNA SAMMSON. Nature. 2016; 531:518-522.

15. Zhu H, Li X, Song Y, Zhang P, Xiao Y, Xing Y. Long noncoding RNA ANRIL is up-regulated in bladder cancer and regulates bladder cancer cell proliferation and apoptosis through the intrinsic pathway. Biochemical and biophysical research communications. 2015; 467:223-228.

16. Zhang J, Shi Z, Nan Y, Li M. Inhibiting malignant phenotypes of the bladder cancer cells by silencing long noncoding RNA SChLAP1. International urology and nephrology. 2016; 48:711-716.

17. Chen M, Zhuang C, Liu Y, Li J, Dai F, Xia M, Zhan Y, Lin J, Chen Z, He A, Xu W, Zhao G, Guo Y, et al. Tetracyclineinducible shRNA targeting antisense long non-coding RNA HIF1A-AS2 represses the malignant phenotypes of bladder cancer. Cancer letters. 2016; 376:155-164.

18. Luo H, Zhao X, Wan X, Huang S, Wu D. Gene microarray analysis of the IncRNA expression profile in human urothelial carcinoma of the bladder. International journal of clinical and experimental medicine. 2014; 7:1244-1254.

19. Liu L, Liu Y, Zhuang C, Xu W, Fu X, Lv Z, Wu H, Mou L, Zhao G, Cai Z, Huang W. Inducing cell growth arrest and apoptosis by silencing long non-coding RNA PCAT-1 in human bladder cancer. Tumour biology. 2015; 36:7685-7689.

20. Chen Z, Liu Y, He A, Li J, Chen M, Zhan Y, Lin J, Zhuang C, Liu L, Zhao G, Huang W, Cai Z. Theophylline controllable RNAi-based genetic switches regulate expression of lncRNA TINCR and malignant phenotypes in bladder cancer cells. Scientific reports. 2016; 6:30798.

21. Ying L, Chen Q, Wang Y, Zhou Z, Huang Y, Qiu F. Upregulated MALAT-1 contributes to bladder cancer cell migration by inducing epithelial-to-mesenchymal transition. Molecular bioSystems. 2012; 8:2289-2294.

22. Han Y, Liu Y, Gui Y, Cai Z. Long intergenic non-coding RNA TUG1 is overexpressed in urothelial carcinoma of the bladder. Journal of surgical oncology. 2013; 107:555-559.
23. Cancer Genome Atlas Research N. Comprehensive molecular characterization of urothelial bladder carcinoma. Nature. 2014; 507:315-322.

24. Di Gesualdo F, Capaccioli S, Lulli M. A pathophysiological view of the long non-coding RNA world. Oncotarget. 2014; 5:10976-10996. doi: 10.18632/oncotarget.2770.

25. Zhu YP, Bian XJ, Ye DW, Yao XD, Zhang SL, Dai B, Zhang HL, Shen YJ. Long noncoding RNA expression signatures of bladder cancer revealed by microarray. Oncol Lett. 2014; 7:1197-1202.

26. Peter S, Borkowska E, Drayton RM, Rakhit CP, Noon A, Chen W, Catto JW. Identification of differentially expressed long noncoding RNAs in bladder cancer. Clinical cancer research. 2014; 20:5311-5321.

27. Hood JD, Cheresh DA. Role of integrins in cell invasion and migration. Nature reviews Cancer. 2002; 2:91-100.

28. Ozes AR, Miller DF, Ozes ON, Fang F, Liu Y, Matei D, Huang T, Nephew KP. NF-kappaB-HOTAIR axis links DNA damage response, chemoresistance and cellular senescence in ovarian cancer. Oncogene. 2016; 35:5350-5361.

29. Liu Z, Sun M, Lu K, Liu J, Zhang M, Wu W, De W, Wang Z, Wang $\mathrm{R}$. The long noncoding RNA HOTAIR contributes to cisplatin resistance of human lung adenocarcinoma cells via downregualtion of p21(WAF1/CIP1) expression. PloS one. 2013; 8:e77293.

30. Plimack ER, Dunbrack RL, Brennan TA, Andrake MD, Zhou Y, Serebriiskii IG, Slifker M, Alpaugh K, Dulaimi E, Palma N, Hoffman-Censits J, Bilusic M, Wong YN, et al. Defects in DNA Repair Genes Predict Response to Neoadjuvant Cisplatin-based Chemotherapy in Muscleinvasive Bladder Cancer. European urology. 2015; 68:959-967.

31. Baras AS, Gandhi N, Munari E, Faraj S, Shultz L, Marchionni L, Schoenberg M, Hahn N, Hoque MO, Berman D, Bivalacqua TJ, Netto G. Identification and Validation of Protein Biomarkers of Response to Neoadjuvant Platinum Chemotherapy in Muscle Invasive Urothelial Carcinoma. PloS one. 2015; 10:e0131245.

32. Tay Y, Rinn J, Pandolfi PP. The multilayered complexity of ceRNA crosstalk and competition. Nature. 2014; 505:344-352.

33. Huang M, Zhong Z, Lv M, Shu J, Tian Q, Chen J. Comprehensive analysis of differentially expressed profiles of lncRNAs and circRNAs with associated co-expression and ceRNA networks in bladder carcinoma. Oncotarget. 2016; 7:47186-47200. doi: 10.18632/oncotarget.9706.

34. Paraskevopoulou MD, Georgakilas G, Kostoulas N, Reczko M, Maragkakis M, Dalamagas TM, Hatzigeorgiou AG. DIANA-LncBase: experimentally verified and computationally predicted microRNA targets on long non-coding RNAs. Nucleic Acids Res. 2013; 41:D239-245.

35. Hao Y, Wu W, Li H, Yuan J, Luo J, Zhao Y, Chen R. NPInter v3.0: an upgraded database of noncoding RNA-associated interactions. Database (Oxford). 2016; 2016: baw057. 
36. Cerami E, Gao J, Dogrusoz U, Gross BE, Sumer SO, Aksoy BA, Jacobsen A, Byrne CJ, Heuer ML, Larsson E, Antipin Y, Reva B, Goldberg AP, et al. The cBio cancer genomics portal: an open platform for exploring multidimensional cancer genomics data. Cancer Discov. 2012; 2:401-404.

37. Gao J, Aksoy BA, Dogrusoz U, Dresdner G, Gross B, Sumer SO, Sun Y, Jacobsen A, Sinha R, Larsson E, Cerami E, Sander C, Schultz N. Integrative analysis of complex cancer genomics and clinical profiles using the cBioPortal. Sci Signal. 2013; 6:11.
38. Kyriazis AA, Kyriazis AP, McCombs WB 3rd, Peterson WD Jr. Morphological, biological, and biochemical characteristics of human bladder transitional cell carcinomas grown in tissue culture and in nude mice. Cancer research. 1984; 44:3997-4005.

39. Ding Y, Chan CY, Lawrence CE. Sfold web server for statistical folding and rational design of nucleic acids. Nucleic Acids Res. 2004; 32:W135-141. 